\title{
Identification of KRAP-expressing cells and the functional relevance of KRAP to the subcellular localization of $\mathrm{IP}_{3} \mathrm{R}$ in the stomach and kidney
}

\author{
TAKAHIRO FUJIMOTO ${ }^{1,2}$ and SENJI SHIRASAWA ${ }^{1,2}$ \\ ${ }^{1}$ Department of Cell Biology, Faculty of Medicine and ${ }^{2}$ Central Research Institute for Advanced Molecular \\ Medicine, Fukuoka University, 7-45-1 Nanakuma, Jonan-ku, Fukuoka 814-0180, Japan
}

Received June 4, 2012; Accepted August 20, 2012

DOI: $10.3892 /$ ijmm.2012.1126

\begin{abstract}
K R A S$-induced actin-interacting protein (KRAP), originally identified as one of the deregulated genes expressed in colorectal cancer, participates under physiological conditions in the regulation of systemic energy homeostasis and of the exocrine system. We have recently found that KRAP is a molecule associated with inositol 1,4,5-trisphosphate receptor $\left(\mathrm{IP}_{3} \mathrm{R}\right)$ and is critical for the proper subcellular localization of $\mathrm{IP}_{3} \mathrm{R}$ in the liver and the pancreas. However, the expression of KRAP and its precise function in other tissues remain elusive. In this study, we aimed to identify the KRAP-expressing cells in mouse stomach and kidneys and to examine the relevance of KRAP expression in the regulation of $\mathrm{IP}_{3} \mathrm{R}$ localization in these tissues. In the stomach, double immunohistochemical staining for KRAP and $\mathrm{IP}_{3} \mathrm{R}$ demonstrated that KRAP was expressed along with the apical regions in the mucous cells and the chief cells, and $\mathrm{IP}_{3} \mathrm{R} 3$ was dominantly co-localized with KRAP in these cells. Furthermore, $\mathrm{IP}_{3} \mathrm{R} 2$ was also co-localized with $\mathrm{IP}_{3} \mathrm{R} 3$ in the chief cells. It is of note that the proper localization of IP $R 3$ and $I_{3} R 2$ in the chief cells and of $I_{3} R 3$ in the mucous cells were significantly abrogated in $K R A P$-deficient mice. In the kidneys, KRAP was expressed in both the apical and the basal regions of the proximal tubular cells. Intriguingly, KRAP deficiency abrogated the localization of $\mathrm{IP}_{3} \mathrm{R} 1$ in the proximal tubular cells. Finally, co-immunoprecipitation study in the stomachs and the kidneys validated the physical association of KRAP with $\mathrm{IP}_{3}$ Rs. These findings demonstrate that KRAP physically associates with $\mathrm{IP}_{3} \mathrm{Rs}$ and regulates the proper localization of IP $\mathrm{I}_{3} \mathrm{Rs}$ in the mucous cells and the chief cells of the stomach and in the proximal tubular cells of the kidneys.
\end{abstract}

Correspondence to: Professor Senji Shirasawa, Department of Cell Biology, Faculty of Medicine, Fukuoka University, 7-45-1 Nanakuma, Jonan-ku, Fukuoka 814-0180, Japan

E-mail: sshirasa@fukuoka-u.ac.jp

Key words: RAS-induced actin-interacting protein, inositol 1,4,5-trisphosphate receptor, immunohistochemical staining, proteinprotein interaction, stomach, kidney

\section{Introduction}

Three inositol 1,4,5-trisphosphate receptor $\left(\mathrm{IP}_{3} \mathrm{R}\right)$ subtypes, $\mathrm{IP}_{3} \mathrm{R} 1, \mathrm{IP}_{3} \mathrm{R} 2$, and $\mathrm{IP}_{3} \mathrm{R} 3$, are differentially expressed among tissues (1-5) and function as the $\mathrm{Ca}^{2+}$ release channel on endoplasmic reticulum membranes (6-10). $\mathrm{IP}_{3} \mathrm{R}$ is regulated by many intracellular modulators, phosphorylation by kinases, and associated proteins (11-15).

$K R A S$-induced actin-interacting protein (KRAP) was originally identified as one of the deregulated expression gene in the colorectal cancer cell line, HCT116 (16). The previous studies using KRAP-knockout (KRAP-KO) mice demonstrate that KRAP participates in the regulation of systemic energy homeostasis (17) and of exocrine system (18). Among the adult mouse tissues, KRAP is ubiquitously expressed, with high levels in the pancreas, liver, and brown adipose tissues, and KRAP localizes in the restricted apical regions of the liver parenchymal cells and of the pancreatic exocrine acinar cells (19). Our recent findings indicate that KRAP associates with $\mathrm{IP}_{3} \mathrm{R}$ to regulate its proper subcellular localization in the mouse liver and the pancreas (20) as well as in immortalized cultured cell lines (21). Despite these advances, it remains largely unknown which cell types express KRAP among the other tissues including stomach and kidneys.

Herein, we performed immunohistological analysis and identified the exact KRAP-expressing cells in the stomach and the kidneys, and demonstrated that KRAP plays critical role in the regulation of the precise subcellular localization of $\mathrm{IP}_{3} \mathrm{R}$ in the mucous and the chief cells of the stomach and in the proximal tubular cells of the kidneys.

\section{Materials and methods}

Animals. All animals used in this study were treated in accordance with the guidelines of Fukuoka University. KRAPknockout mice were generated as described previously (17).

Immunohistochemical staining. Immunohistochemical staining was performed as described previously $(19,20)$. Specific signals were detected by using rabbit polyclonal anti-KRAP antibody (19), mouse monoclonal anti-ZO-1 antibody (ZYMED), mouse monoclonal anti-IP ${ }_{3} \mathrm{R} 3$ antibody (610313) from BD Transduction 


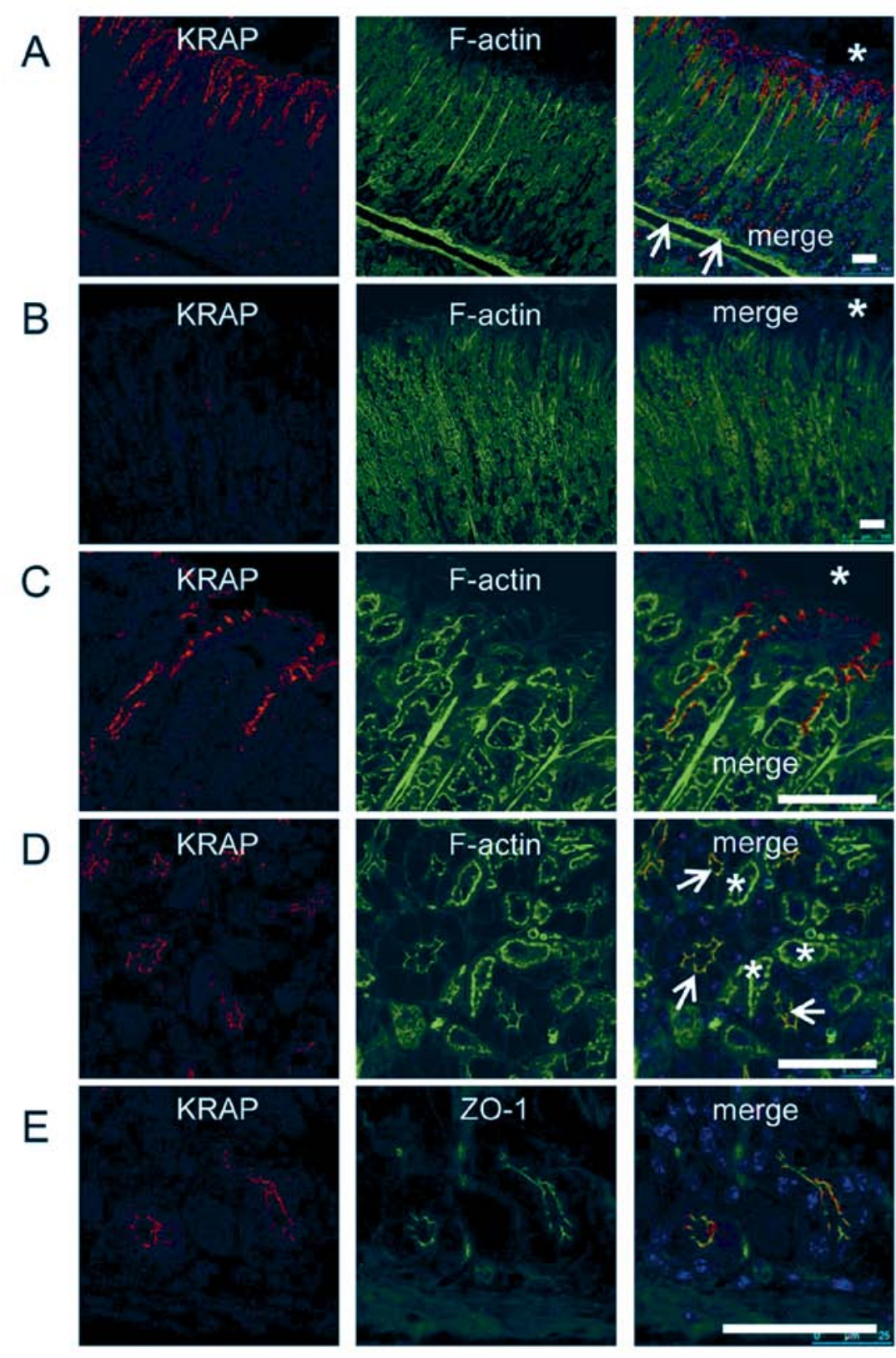

Figure 1. KRAP expression in the mucous cells and the chief cells of the mouse stomach. (A-D) Fluorescent confocal images of stomach sections for KRAP (red), filamentous actin (F-actin) with phalloidin (green), and the merged photo. Low magnification images from the pit region to the base region of gastric glands from wild-type (A) or KRAP-deficient (B) mice. Asterisk and arrows indicate gastric lumen and muscularis mucosae beneath the base region, respectively. (C) High magnification images of the pit region of gastric glands. Asterisk indicates gastric lumen. (D) High magnification images of the base regions of gastric glands. Asterisks and arrowheads indicate the parietal cells and the apical membranes of the chief cells, respectively. (E) Fluorescent confocal images of the base regions of gastric glands for KRAP (red), ZO-1 (green), and the merged photo. Blue, 4',6-diamidino-2-phenylindole (DAPI) staining; scale bar, $50 \mu \mathrm{m}$.

Laboratories, rabbit polyclonal anti-IP ${ }_{3} \mathrm{R} 2$ antibody $(\mathrm{AB} 3000)$ from Millipore, and rabbit polyclonal anti-IP ${ }_{3} \mathrm{R} 1$ antibody (ab5840) from Abcam.

Immunoprecipitations and western blotting. Immunoprecipitations and western blotting were performed as described previously $(19,20)$.

\section{Results}

Localization of KRAP protein in the adult mouse stomach. To examine the cellular distribution of KRAP protein in the adult mouse tissues, we performed immunohistochemical staining by using anti-KRAP antibody. In the stomach, strong KRAP immunoreactivity was restricted to the pit regions of gastric glands (Fig. 1A), whereas significant expression of KRAP was not detected in the muscularis mucosae beneath the gastric glands (Fig. 1A, arrows). The specificity of KRAP expression in the stomach was confirmed by using $K R A P-\mathrm{KO}$ tissue as a control (Fig. 1B). In the pit region of the gastric gland, where columnar surface mucous cells mainly exist (22), KRAP was localized beneath the apical membranes of the mucous cells (Fig. 1C). In the base region of the gastric glands, where zymogenic chief cells mainly exist, coronal plane of deeper gastric 

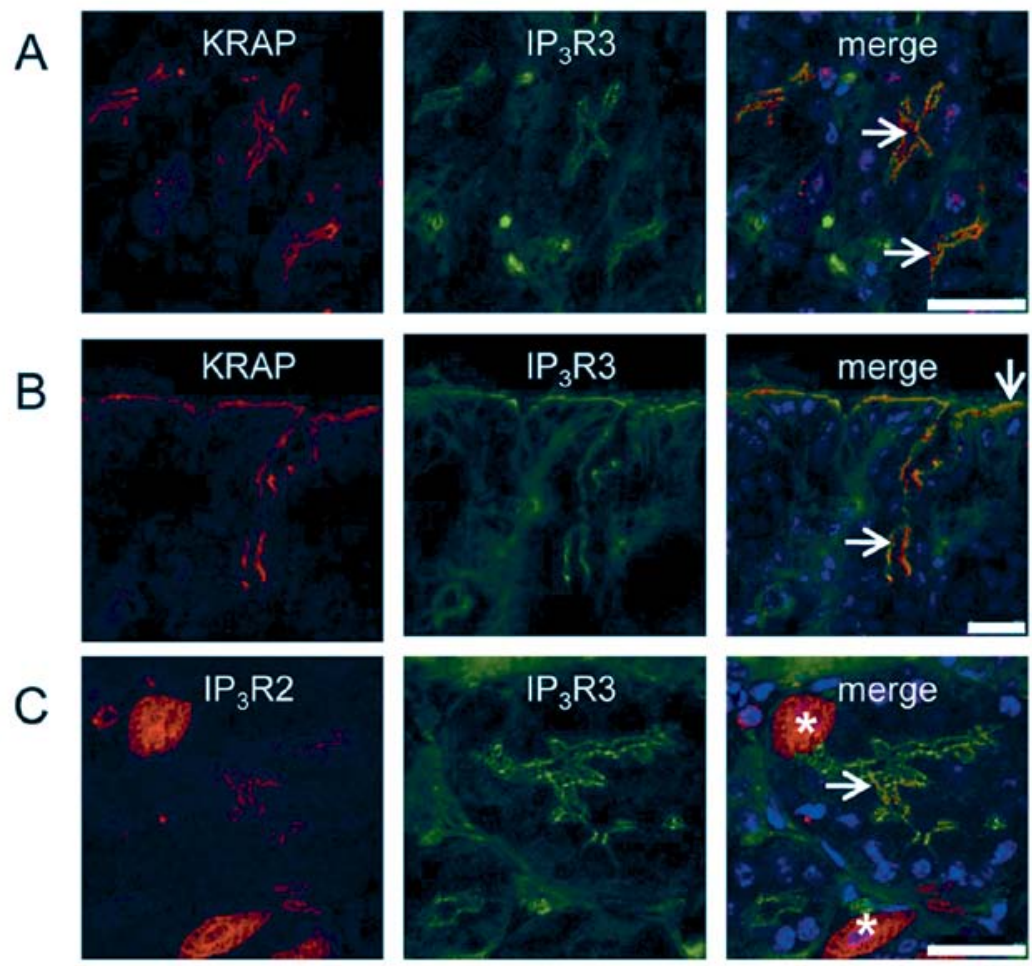

$\mathrm{D}$
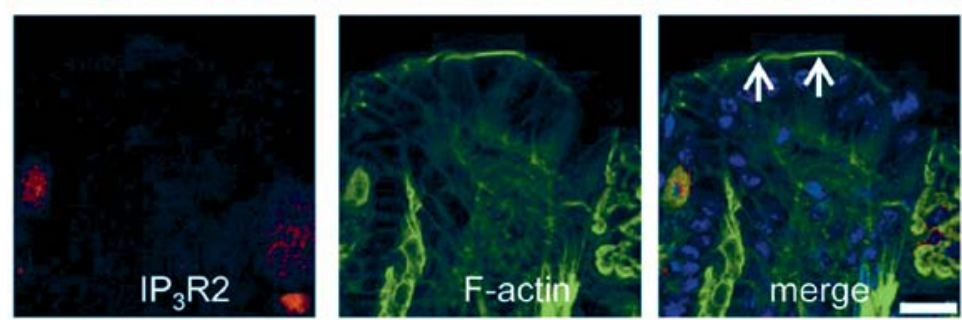

Figure 2. Colocalization of KRAP with $\mathrm{IP}_{3}$ Rs in the chief cells and the mucous cells of the mouse stomach. (A) Fluorescent confocal images of the base region of gastric glands for KRAP (red), $\mathrm{IP}_{3} \mathrm{R} 3$ (green), and the merged photo. Arrows indicate the apical membranes of the chief cells. (B) Fluorescent confocal images of the pit region of gastric glands for KRAP (red), $\mathrm{IP}_{3} \mathrm{R} 3$ (green), and the merged photo. Arrows indicate the apical membranes of the mucous cells. (C) Fluorescent confocal images of the base region of gastric glands for $\mathrm{IP}_{3} \mathrm{R} 2$ (red), $\mathrm{IP}_{3} \mathrm{R} 3$ (green), and the merged photo. Asterisks and arrow indicate the parietal cells and the apical membranes of the chief cells, respectively. (D) Fluorescent confocal images of the pit region of gastric glands for IP ${ }_{3} R 2$ (red), IP $P_{3} R 3$ (green), and the merged photo. Arrows indicate the apical membranes of the mucous cells. Blue, 4',6-diamidino-2-phenylindole (DAPI) staining; scale bar, $25 \mu \mathrm{m}$.

glands showed that KRAP was restricted to the apical regions of the chief cells (Fig. 1D, arrowheads), whereas KRAP was not detected in the parietal cells (Fig. 1D, asterisks). The distinction between the chief and the parietal cells was validated by ZO-1 staining as described (23), indicating that KRAP was expressed in the ZO-1-positive chief cells but not in the ZO-1-negative parietal cells (Fig. 1E).

$K R A P$ co-localized with $I P_{3} R$ in the stomach. Since we previously reported that KRAP associates with particular subtypes of $I_{3} R$ in the liver and the pancreas (20), we examined whether KRAP in the stomach is also co-localized with $\mathrm{IP}_{3} \mathrm{R}$. Double-immunostaining of the stomach for KRAP and $\mathrm{IP}_{3} \mathrm{R} 3$ revealed that KRAP was co-localized with $\mathrm{IP}_{3} \mathrm{R} 3$ in the apical regions of both the chief cells (Fig. 2A, arrows) and the mucous cells (Fig. 2B, arrows). Of note, $\mathrm{IP}_{3} \mathrm{R} 2$ co-existed with $\mathrm{IP}_{3} \mathrm{R} 3$ in the chief cells (Fig. 2C, arrow) but not in the parietal cells (Fig. 2C, asterisks). Furthermore, $\mathrm{IP}_{3} \mathrm{R} 2$ was not detected in the mucous cells (Fig. 2D, arrows). These results indicated that KRAP was co-localized with $\mathrm{IP}_{3} \mathrm{R} 2$ and $\mathrm{IP}_{3} \mathrm{R} 3$ in the chief cells and with $\mathrm{IP}_{3} \mathrm{R} 3$ in the mucous cells.
Impaired localization of $I P_{3} R$ in the KRAP-deficient chief cells and the mucous cells. We addressed the functional relevance of KRAP to the proper localization of $\mathrm{IP}_{3} \mathrm{R}$ by using $K R A P-\mathrm{KO}$ mice. $\mathrm{IP}_{3} \mathrm{R} 3$ was located in the apical region of the chief cells (Fig. 3A, arrow) and of the mucous cells (Fig. 3C, arrows) in the wild-type (WT) mouse stomach, whereas the restricted localization of $\mathrm{IP}_{3} \mathrm{R} 3$ appeared to be diminished in the KRAP-KO stomach (Fig. 3B, arrow; 3D, arrows). Furthermore, $\mathrm{IP}_{3} \mathrm{R} 2$ was detected in both the chief cells (Fig. 3E, arrows) and the parietal cells (Fig. 3E, asterisks) in the WT stomach, whereas the localization of $\mathrm{IP}_{3} \mathrm{R} 2$ in the $K R A P-\mathrm{KO}$ stomach was impaired in the chief cells (Fig. 3F, arrows) but not in the parietal cells (Fig. 3F, asterisks). Thus, KRAP plays critical role in the regulation of the proper localization of $\mathrm{IP}_{3} \mathrm{R} 2$ and $\mathrm{IP}_{3} \mathrm{R} 3$ in the chief cells and of $\mathrm{IP}_{3} \mathrm{R} 3$ in the mucous cells.

KRAP expression and its contribution to the localization of $I P_{3} R 1$ in the proximal tubules of the mouse kidney. To examine the cellular distribution of KRAP protein in the adult mouse kidneys, we performed immunohistochemical staining by 

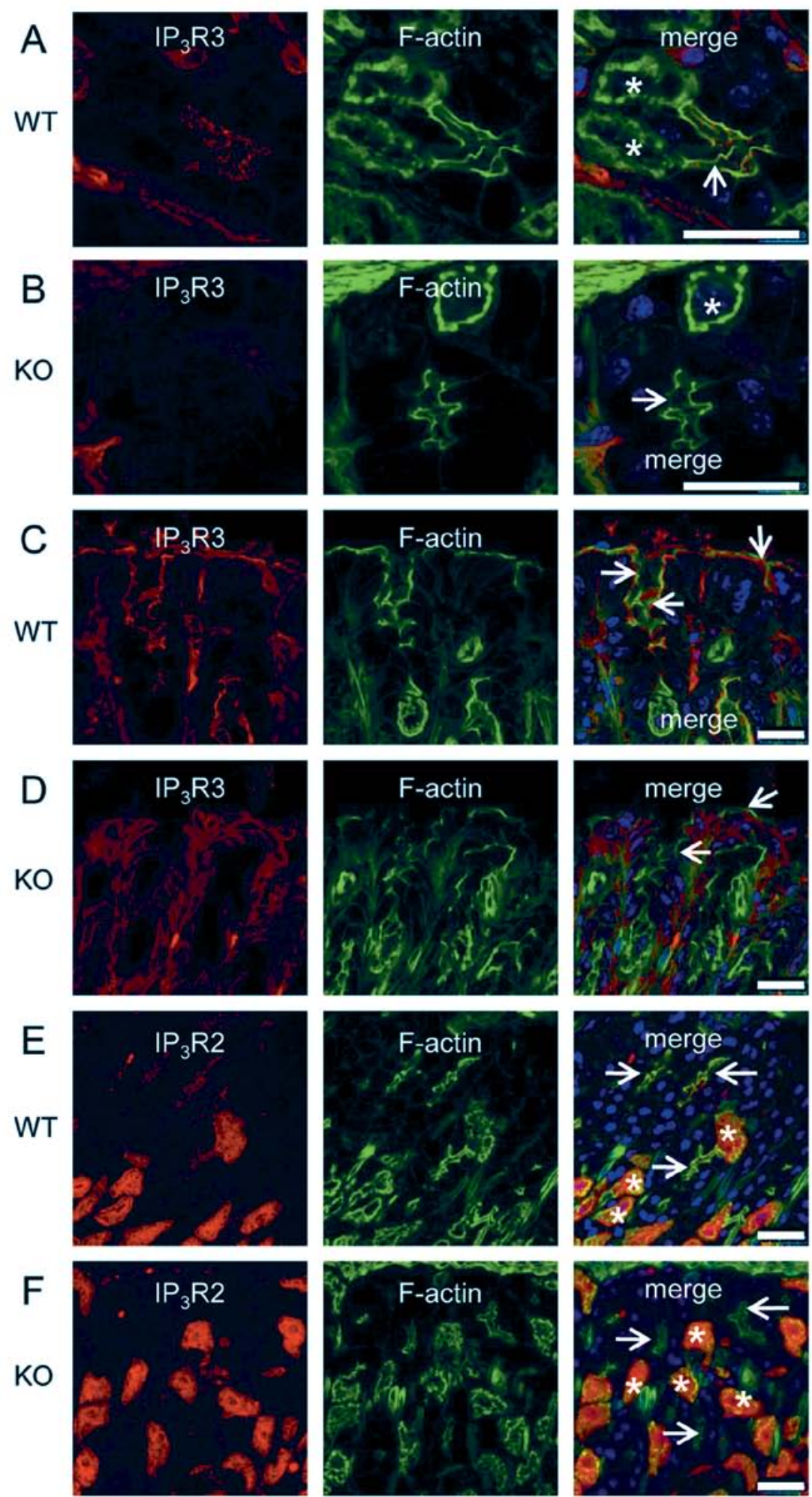

Figure 3. Impaired localization of $\mathrm{IP}_{3} \mathrm{Rs}$ in the $K R A P$-deficient chief cells and the mucous cells. (A and B) Fluorescent confocal images of the base region of gastric glands for $\mathrm{IP}_{3} \mathrm{R} 3$ (red), F-actin with phalloidin (green), and the merged photo from wild-type (WT) (A) or KRAP-deficient (KO) (B) mice. Asterisks and arrows indicate the parietal cells and the apical membranes of the chief cells, respectively. (C and D) Fluorescent confocal images of the pit region of gastric glands for $\mathrm{IP}_{3} \mathrm{R} 3$ (red), F-actin (green), and the merged photo from WT (C) or KO (D) mice. Arrows indicate the apical membranes of the mucous cells. (E and F) Fluorescent confocal images of the base region of gastric glands for IP ${ }_{3} \mathrm{R} 2$ (red), F-actin (green), and the merged photo from WT (E) or KO (F) mice. Asterisks and arrows indicate the parietal cells and the apical membranes of the chief cells, respectively. Blue, 4',6-diamidino-2-phenylindole (DAPI) staining; scale bar, $25 \mu \mathrm{m}$.

using anti-KRAP antibody. The specificities of the signals were validated by comparing the immunoreactivities of WT and KRAP-KO mouse tissues. In the WT kidneys, intense immunoreactivities were observed in the renal proximal tubules (Fig. 4A) but not in the renal distal tubules (data not shown). On the other hand, significant immunoreactive signal was 

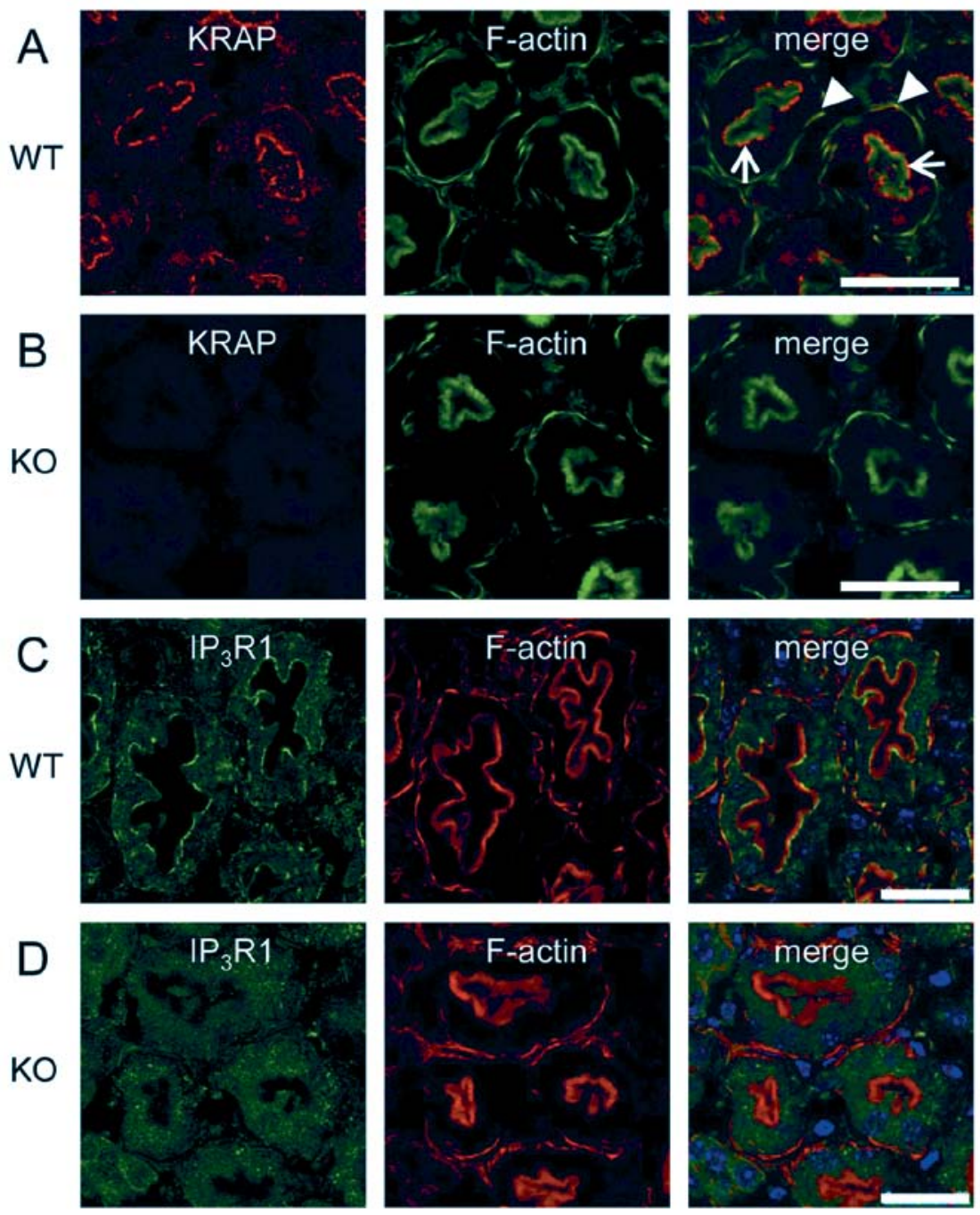

Figure 4. KRAP expression and its contribution to the localization of $\mathrm{IP}_{3} \mathrm{R} 1$ in the proximal tubules of the mouse kidney. (A and $\left.\mathrm{B}\right)$ Fluorescent confocal images of the proximal tubules of kidney for KRAP (red), F-actin (green), and the merged photo from wild-type (WT) (A) or KRAP-deficient (KO) (B) mice. Arrowheads and arrows indicate the basolateral and the apical regions of the proximal tubules, respectively. (C and D) Fluorescent confocal images of the proximal tubules of kidney for $\mathrm{IP}_{3} \mathrm{R} 1$ (green), F-actin (red), and the merged photo from WT (C) or KO (D) mice. Blue, 4',6-diamidino-2-phenylindole (DAPI) staining; scale bar, $25 \mu \mathrm{m}$.

not detected in the proximal tubules in the KRAP-KO mice (Fig. 4B). Taken together, these results indicate that KRAP was exactly expressed in the proximal tubules. The proximal tubules were identified by the presence of the brush-border stained with phalloidin (Fig. 4A and B). Immunostaining in the proximal region showed that KRAP was accumulated beneath the brush-border (Fig. 4A, arrows) and KRAP was also detected in the basolateral actin bundles (Fig. 4A, arrowheads). We next examined which subtypes of $\mathrm{IP}_{3} \mathrm{R}, \mathrm{IP}_{3} \mathrm{R} 1$, $\mathrm{IP}_{3} \mathrm{R} 2$, and $\mathrm{IP}_{3} \mathrm{R} 3$, expressed in the proximal tubular cells, revealing that $I_{3} R 1$ (Fig. $4 C$ ) but not $I_{3} R 2$ or $I_{3} R 3$ (data not shown) was detected in the beneath the brush-border and in the basolateral actin bundles. Finally, we addressed the functional relevance of KRAP expression in the proximal tubular cells to the regulation of $\mathrm{IP}_{3} \mathrm{R}$ localization. It is of note that the restricted localization of $\mathrm{IP}_{3} \mathrm{R} 1$ detected in the WT mouse kidney (Fig. 4C) was disturbed in the KRAP-KO mouse kidney (Fig. 4D). Thus, KRAP plays critical role in the regulation of the proper localization of $I_{3} R 1$ in the proximal tubular cells.
KRAP interacts with $I P_{3} R 1$ in the kidneys and with $I P_{3} R 3$ in the stomach. As described above, immunohistochemical signals for particular $\mathrm{IP}_{3} \mathrm{R}$ subtypes in the KRAP-KO mouse kidneys or the stomach were abrogated, leading us to check the expression levels of $\mathrm{IP}_{3} \mathrm{R}$ between the WT and KRAP-KO mouse tissues. Normal expression levels of $I_{3} R 1$ and $I_{3} R 3$ were detected in the $K R A P-\mathrm{KO}$ mouse kidney and the stomach, respectively, compared with the WT mouse tissues (Fig. 5A), suggesting that mislocalizations but not deregulated expressions of $\mathrm{IP}_{3} \mathrm{R}$ occur in the $K R A P-\mathrm{KO}$ mouse kidneys and the stomach. Next, to examine the physical association of KRAP with $\mathrm{IP}_{3} \mathrm{R}$, we performed co-immunoprecipitations by anti-KRAP antibody in the kidneys or the stomach, in which we could not evaluate the specific association of $\mathrm{IP}_{3} \mathrm{R} 2$ with KRAP due to lack of $\mathrm{IP}_{3} \mathrm{R} 2$-specific antibody available for western blotting. In the preparations from the WT mouse tissues, KRAP precipitates $\mathrm{IP}_{3} \mathrm{R} 1$ and $\mathrm{IP}_{3} \mathrm{R} 3$ in the kidney and the stomach, respectively (Fig. 5B). The specificity of co-immunoprecipitations of $\mathrm{IP}_{3} \mathrm{R}$ was confirmed by using KRAP-KO mouse tissue as a control (Fig. 5B). Thus, KRAP 
A

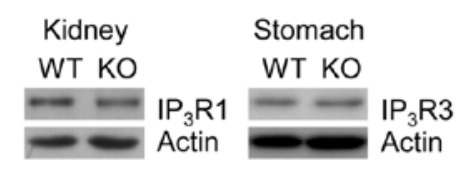

B

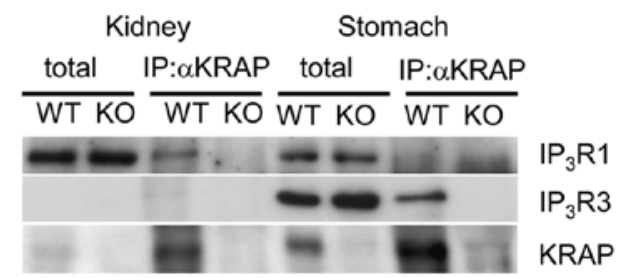

Figure 5.KRAP interacts with $\mathrm{IP}_{3} \mathrm{R} 1$ in the kidney and with $\mathrm{IP}_{3} \mathrm{R} 3$ in the stomach. (A) Western blots showing comparable expression levels of $I_{3} R 1$ in the kidney (left) or of $\mathrm{IP}_{3} \mathrm{R} 3$ in the stomach (right) between $K R A P$-deficient (KO) and wild-type (WT) mice. (B) Anti-KRAP ( $\alpha$ KRAP) immunoprecipitations were performed using mouse kidneys and stomachs from WT or KO mice, followed by western blotting with anti-KRAP, anti-IP ${ }_{3} \mathrm{R} 1$, or anti$\mathrm{IP}_{3} \mathrm{R} 3$ antibodies. total, total lysate;IP, immunoprecipitation; $\alpha$, anti-.

physically interacts with $\mathrm{IP}_{3} \mathrm{R} 1$ in the kidneys and with $\mathrm{IP}_{3} \mathrm{R} 3$ in the stomach.

\section{Discussion}

In this study, we demonstrated that KRAP protein expression and the subcellular localization was restricted beneath the apical and/or basolateral membranes in specific cell types of the stomach and the kidneys, in which KRAP physically associated with particular $\mathrm{IP}_{3} \mathrm{R}$ subtype(s). In the $K R A P-\mathrm{KO}$ mouse stomach and the kidneys, the polarized localization of $\mathrm{IP}_{3} \mathrm{R}$ was impaired, indicating that KRAP plays critical roles in the regulation of the proper subcellular localization of $\mathrm{IP}_{3} \mathrm{R}$ in the stomach and the kidneys.

Notably, KRAP as well as $\mathrm{IP}_{3} \mathrm{R} 3$ proteins were polarized beneath the apical membranes facing the gastric gland lumen and were absent in the parietal cells (Fig. 1), suggesting an association of these proteins with chief cell functions including pepsinogen secretion (22-24). From this view point, KRAP expression and the localization beneath the apical membranes of the pancreatic acinar cells (19), another type of zymogen cells, may suggest a similar role for KRAP in the stomach and the pancreas. Considering the fact that KRAP physically interacts with $\mathrm{IP}_{3} \mathrm{R}$ to regulate its proper localization in these tissues, stomach (Figs. 2, 3 and 5) and pancreas (20), and that double-knockout of $\mathrm{IP}_{3} \mathrm{R} 2$ and $\mathrm{IP}_{3} \mathrm{R} 3$ in mice revealed a failure in secretory function in the pancreas (25), KRAP seems to be involved in the exocrine systems. Actually, the pancreatic acinar cells in KRAP-KO mice showed an increased amount of zymogen granules, although they seemed to maintain the proper physiological agonist-induced exocytosis (18). Thus, exact functional relevance of KRAP and its interaction with $\mathrm{IP}_{3} \mathrm{R}$ to the exocrine systems in the pancreas and the stomach should await future studies.

It is of note that KRAP was restricted to both the apical region and the basolateral region of the proximal tubular cells of the kidneys (Fig. 4), and that KRAP physically associated with $\mathrm{IP}_{3} \mathrm{R} 1$ in the kidneys (Fig. 5). Furthermore, our previous study showed that KRAP was distributed along the bile canaliculi of hepatocytes and underneath the apical membrane of pancreatic acinar cells (19). All these KRAP localizations in the distinct tissues examined are restricted to epithelial cell types bearing well-developed cell polarity, cell-cell junction and microvilli, where transports of various substances between epithelial cells and extracellular spaces, exocrine space or blood stream occur $(22,26-28)$. Since $K R A P-\mathrm{KO}$ mice displayed profound metabolic disorders after birth without developmental defects, and certain systemic inter-tissue dysregulations appeared to underlie the metabolic phenotypes (17), KRAP might play physiological roles in secretion and/or absorption functions after birth rather than in developmental events.

Renal proximal tubules serve the reabsorption of the bulk of substances filtered in the glomeruli and the excretion $(26,29)$. These two opposite transports are accomplished by the coordinated action of ion channels and transporters located in the brush border membrane and basolateral membrane (29-31). Thus, the polarized expression of these membrane proteins is crucial for the function of the proximal tubules. Based on the findings that KRAP protein possesses characteristic features like scaffolding protein, such as polarized localization and transporting of $\mathrm{IP}_{3} \mathrm{R}$, potential functional relevance of KRAP to these processes would be suspected.

In conclusion, we identified the exact KRAP-expressing cells in the stomach and the kidneys, and found that KRAP physically associates with $\mathrm{IP}_{3} \mathrm{R}$ to regulate its proper subcellular localization in vivo. Considering the KRAP function as an $\mathrm{IP}_{3} \mathrm{R}$ regulator and the importance of KRAP in energy homeostasis in vivo, further research on the exact relevance of the association between KRAP and $\mathrm{IP}_{3} \mathrm{R}$ to the biological phenomena will lead to a better understanding of physiological metabolic processes.

\section{Acknowledgements}

This work was supported in part by the Ministry of Education, Culture, Sports, Science and Technology (MEXT)-Supported Program for the Strategic Research Foundation at Private Universities, a Grant-in-Aid for Scientific Research from the Japan Society for the Promotion of Science. We thank Takami Danno and Yoko Tanaka for their technical assistance.

\section{References}

1. Ross CA, Danoff SK, Schell MJ, Snyder SH and Ullrich A: Three additional inositol 1,4,5-trisphosphate receptors: molecular cloning and differential localization in brain and peripheral tissues. Proc Natl Acad Sci USA 89: 4265-4269, 1992.

2. Sharp AH, McPherson PS, Dawson TM, Aoki C, Campbell KP and Snyder SH: Differential immunohistochemical localization of inositol 1,4,5-trisphosphate- and ryanodine-sensitive $\mathrm{Ca}^{2+}$ release channels in rat brain. J Neurosci 13: 3051-3063, 1993.

3. Sugiyama T, Yamamoto-Hino M, Miyawaki A, Furuichi T, Mikoshiba K and Hasegawa M: Subtypes of inositol 1,4,5-trisphosphate receptor in human hematopoietic cell lines: dynamic aspects of their cell-type specific expression. FEBS Lett 349: 191-196, 1994.

4. Newton CL, Mignery GA and Südhof TC: Co-expression in vertebrate tissues and cell lines of multiple inositol 1,4,5-trisphosphate (InsP3) receptors with distinct affinities for InsP3. J Biol Chem 269: 28613-28619, 1994.

5. Wojcikiewicz RJ: Type I, II, and III inositol 1,4,5-trisphosphate receptors are unequally susceptible to down-regulation and are expressed in markedly different proportions in different cell types. J Biol Chem 270: 11678-11683, 1995. 
6. Jayaraman T, Ondriasová E, Ondrias K, Harnick DJ and Marks AR: The inositol 1,4,5-trisphosphate receptor is essential for T-cell receptor signaling. Proc Natl Acad Sci USA 92 6007-6011, 1995.

7. Khan AA, Soloski MJ, Sharp AH, Schilling G, Sabatini DM, Li SH, Ross CA and Snyder SH: Lymphocyte apoptosis: mediation by increased type 3 inositol 1,4,5-trisphosphate receptor. Science 273: 503-507, 1996.

8. Sugawara H, Kurosaki M, Takata M and Kurosaki T: Genetic evidence for involvement of type 1, type 2 and type 3 inositol 1,4,5-trisphosphate receptors in signal transduction through the B-cell antigen receptor. EMBO J 16: 3078-3088, 1997.

9. Scharenberg AM, Humphries LA and Rawlings DJ: Calcium signalling and cell-fate choice in B cells. Nat Rev Immunol 7: 778-789, 2007.

10. deSouza N, Cui J, Dura M, McDonald TV and Marks AR: A function for tyrosine phosphorylation of type 1 inositol 1,4,5-trisphosphate receptor in lymphocyte activation. J Cell Biol 179: 923-934, 2007.

11. Patterson RL, Boehning D and Snyder SH: Inositol 1,4,5-trisphosphate receptors as signal integrators. Annu Rev Biochem 73 437-465, 2004

12. Bezprozvanny I: The inositol 1,4,5-trisphosphate receptors. Cell Calcium 38: 261-272, 2005.

13. Foskett JK, White C, Cheung KH and Mak DO: Inositol trisphosphate receptor $\mathrm{Ca}^{2+}$ release channels. Physiol Rev 87: 593-658, 2007.

14. Mikoshiba K: IP3 receptor/ $\mathrm{Ca}^{2+}$ channel: from discovery to new signaling concepts. J Neurochem 102: 1426-1446, 2007.

15. Zhang S, Fritz N, Ibarra C and Uhlén P: Inositol 1,4,5-trisphosphate receptor subtype-specific regulation of calcium oscillations. Neurochem Res 36: 1175-1185, 2011.

16. Inokuchi J, Komiya M, Baba I, Naito S, Sasazuki T and Shirasawa S: Deregulated expression of KRAP, a novel gene encoding actin-interacting protein, in human colon cancer cells. J Hum Genet 49: 46-52, 2004

17. Fujimoto T, Miyasaka K, Koyanagi M, Tsunoda T, Baba I, Doi K, Ohta M, et al: Altered energy homeostasis and resistance to dietinduced obesity in KRAP-deficient mice. PLoS One 4: e4240, 2009.

18. Miyasaka K, Fujimoto T, Kawanami T, Takiguchi S, Jimi A, Funakoshi A and Shirasawa S: Pancreatic hypertrophy in Ki-rasinduced actin-interacting protein gene knockout mice. Pancreas 40: 79-83, 2011.

19. Fujimoto T, Koyanagi M, Baba I, Nakabayashi K, Kato N Sasazuki T and Shirasawa S: Analysis of KRAP expression and localization, and genes regulated by KRAP in a human colon cancer cell line. J Hum Genet 52: 978-984, 2007.
20. Fujimoto T, Machida T, Tanaka Y, Tsunoda T, Doi K, Ota T, Okamura $\mathrm{T}$, et al: KRAS-induced actin-interacting protein is required for the proper localization of inositol 1,4,5-trisphosphate receptor in the epithelial cells. Biochem Biophys Res Commun 407: 438-443, 2011.

21. Fujimoto T, Machida T, Tsunoda T, Doi K, Ota T, Kuroki M and Shirasawa S: Determination of the critical region of KRASinduced actin-interacting protein for the interaction with inositol 1,4,5-trisphosphate receptor. Biochem Biophys Res Commun 408: 282-286, 2011.

22. Mills JC, Andersson N, Stappenbeck TS, Chen CC and Gordon JI: Molecular characterization of mouse gastric zymogenic cells. J Biol Chem 278: 46138-46145, 2003.

23. Zhu L, Hatakeyama J, Zhang B, Makdisi J, Ender C and Forte JG: Novel insights of the gastric gland organization revealed by chief cell specific expression of moesin. Am J Physiol Gastrointest Liver Physiol 296: G185-G195, 2009.

24. Schubert ML: Gastric exocrine and endocrine secretion. Curr Opin Gastroenterol 25: 529-536, 2009.

25. Futatsugi A, Nakamura T, Yamada MK, Ebisui E, Nakamura K, Uchida K, Kitaguchi T, et al: IP3 receptor types 2 and 3 mediate exocrine secretion underlying energy metabolism. Science 309: 2232-2234, 2005

26. Wright SH and Dantzler WH: Molecular and cellular physiology of renal organic cation and anion transport. Physiol Rev 84 987-1049, 2004.

27. Alrefai WA and Gill RK: Bile acid transporters: structure, function, regulation and pathophysiological implications. Pharm Res 24: 1803-1823, 2007.

28. Husain S and Thrower E: Molecular and cellular regulation of pancreatic acinar cell function. Curr Opin Gastroenterol 25: 466-471, 2009.

29. Anzai N, Jutabha P, Kanai Y and Endou H: Integrated physiology of proximal tubular organic anion transport. Curr Opin Nephrol Hypertens 14: 472-479, 2005.

30. Hernando N, Wagner CA, Gisler SM, Biber J and Murer H: PDZ proteins and proximal ion transport. Curr Opin Nephrol Hypertens 13: 569-574, 2004.

31. Brône B and Eggermont J: PDZ proteins retain and regulate membrane transporters in polarized epithelial cell membranes. Am J Physiol Cell Physiol 288: C20-C29, 2005. 